\title{
Tank Reactor Temperature Control using Quantitative Feedback Theory
}

\author{
Veronica Olesen ${ }^{*, * *}$ Claes Breitholtz* Torsten Wik* \\ * Automatic Control, Department of Signals and Systems, \\ Chalmers University of Technology, Göteborg, Sweden \\ (e-mail: veronica@chalmers.se,claesbr@chalmers.se,tw@chalmers.se) \\ ** Solvina, Gruvgatan 37, Västra Frölunda, Sweden \\ (e-mail: veronica.olesen@solvina.se)
}

\begin{abstract}
Temperature control of tank reactors with exothermic reactions is an acknowledgedly difficult task because of complex interacting phenomena, several nonlinearities and unstable dynamics. Traditionally, simple control functions are implemented, implying poor control performance and small operating windows. However, many advanced control schemes have been published, though in many applications they rely on too simplistic process models. Here, a control strategy using only a small number of linear controllers has successfully been applied to a rigorous reactor model, including the cooling system dynamics. The methodology is to first linearize the model in steady-state and relevant non-stationary points and let the resulting models define uncertainties in the frequency plane. Then Quantitative Feedback Theory (QFT) is used to ensure that robust feedback system specifications are fulfilled for all these uncertainties.
\end{abstract}

Keywords: Feedback; temperature control; nonlinear; Nichols diagrams.

\section{INTRODUCTION}

An exothermic reaction is a highly nonlinear and normally unstable process. Thus, efficient control is necessary and also nontrivial. In the present paper, a system with a temperature dependent first order, $A \rightarrow B$, exothermic reaction in an ideally stirred tank reactor operating continuously (CSTR) is studied. Since the reaction is exothermic, a cooler is used for temperature control of the reactor. The cooling system is built up by tubes, located inside the reactor vessel. In Olesen et al. (2008) a nonlinear model of this process is formulated and linearized in a number of operating points. The resulting linearized models have large parameter variations. Further, some models are stable, while others have one or two unstable eigenvalues, reflecting the local instability of the original nonlinear model. Nevertheless, it would be desirable to have the same control strategy for as many operating conditions of the tank reactor system as possible.

Several different control strategies for similar tank reactor models have been published. However, the models in many of these publications are too simplistic, especially with respect to the control input signal. In addition to that, the suggested control strategies may in many cases be too complex to implement in an existing tank reactor process. As an example, in Henson and Seborg (1997) a very simple model of a first order reaction in a CSTR, where the control input is modelled as the temperature of the cooling system, is studied. The model is controlled by a nonlinear controller designed by input/output linearization and a comparison is made with a linear controller, based on model predictive control theory. In that example, both controllers were able to control the model from an unstable steady state point to a reference point representing another unstable steady state as well as to a stable point. However, the nonlinear controller performed best, especially in bringing the model to operate in an open loop unstable operating point. It should be noted that the tank reactor model is only used as an application example. The model is probably too simplistc if the nonlinear control strategy based on input/output linearization is to be used on a true system or on a more complex model. The same model is also used as an example by Morningred et al. (1992) to test an adaptive controller in a discretetime nonlinear predictive control framework. Exactly the same mathematical model is later also used in Zhang and Guay (2005) but to represent a slightly more complex reaction. There, the model is controlled by an adaptive method, combining Lyapunov's stability design, adaptive backstepping and neural network approximation. The use of the mean temperature of the cooling system as control signal implies that the cooling system dynamics is ignored, which can entirely alter the stability properties (see Russo and Bequette (1996)).

A simplified model of a first order reaction in a CSTR is also used by Lagerberg and Breitholtz (1997). They have used a more sophisticated model of the cooling system and discuss both coolant inlet temperature and coolant flow as control signals. The model is controlled by gain scheduling using a series of five linear state feedback controllers based on linear quadratic optimization. The performance for coolant inlet temperature as control signal is good. However, if coolant flow is used as an input, the system becomes unstable for large reference variations, due to the additional nonlinearity arising from the use of coolant flow as control signal. 
The tank reactor model used in the present work is based on a complex general model described in Olesen et al. (2008), where the coolant flow is the control signal. The goal has not been to find an optimal nonlinear controller for this system, but to investigate the possibilities to find a simple control strategy, where a small number of linear controllers are sufficient to control the nonlinear system over a wide temperature interval. The method used for this investigation is quantitative feedback theory (QFT), a method for linear uncertain feedback systems. Here, the nonlinear reactor model is presumed to have no uncertainties, but the linearization in stationary and nonstationary operating points gives a set of linear models that is regarded as an uncertainty description.

\section{QUANTITATIVE FEEDBACK THEORY}

QFT is a well established engineering design philosophy for uncertain feedback problems, see Horowitz (1993) and references therein. The method does not provide an algorithm for design of a predefined control structure, but it provides a framework for finding a suitable controller.

The first step in QFT is to define the plant uncertainties. The uncertainties are used to determine a set of linear plant transfer functions $\left\{P_{i}(s)\right\}$. One arbitrary chosen transfer function is assigned as the nominal model, $P_{\text {nom }}(s)$. As the set of transfer functions has been determined, the value sets $\left\{P_{i}\left(j \omega_{k}\right)\right\}$ are calculated at wisely chosen frequencies, $\omega_{k}$. These value sets are the templates, describing the plant uncertainty at the specified frequencies.

The second step is to define closed loop specifications in the frequency domain, such as $\|S\|_{\infty}<k$, where $S$ is the sensitivity function.

The third step is a computation of Horowitz-Sidi bounds from the templates, the nominal plant and the closed loop specifications at the frequencies $\omega_{k}$. These bounds define areas in a Nichols diagram, where the nominal open loop transfer function, $L_{n o m}(s)=P_{n o m}(s) F_{y}(s)$, cannot pass if the closed loop specifications should hold for all open loop transfer functions $L_{i}(s)=P_{i}(s) F_{y}(s)$ in the set. Calculation of these bounds is very time consuming to perform without computer aid. In this work the Qsyn toolbox for Matlab has been used, Gutman (1996).

In the fourth step of QFT, the feedback controller $F_{y}(s)$ is designed. Classical loop shaping is used to design $F_{y}$ such that the nominal loop transfer does not violate the Horowitz-Sidi bounds in the Nichols chart. After that, it is necessary to check closed loop stability for all plant cases and the $F_{y}$ chosen.

Finally a pre-filter $F_{r}$ can be designed to achieve desired servo specifications.

\section{THE NONLINEAR TANK REACTOR MODEL}

The model used in this article describes an ideally stirred tank reactor operating continuously. If the assumption of ideal stirring would not apply, the reactor model could be divided into compartments as in Olesen et al. (2005). In the reactor a first order exothermic reaction $A \rightarrow B$ is assumed to take place in a liquid solvent (denoted by

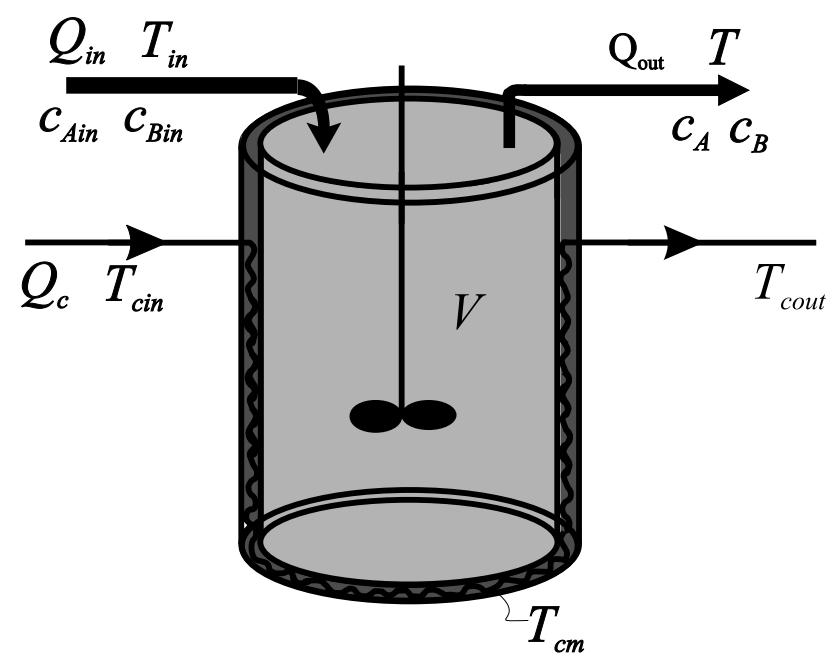

Fig. 1. Sketch of the tank reactor. Control signal is coolant flow, $Q_{c}$, disturbances are changes in continuous inflow parameters, $Q_{i n}, T_{i n}, c_{A i n}, c_{B i n}$ and variations in inflow temperature of the coolant, $T_{c i n}$. State variables are the concentrations, $c_{A}$ and $c_{B}$ in the reactor, reactor temperature $T$ and the mean temperature of the cooling system, $T_{c m}$. The reactor temperature is also the measured output signal.

subscript $l$ ). A cooler, built up by tubes situated inside the reaction vessel, is used for the temperature control of the reactor. Through these tubes cooling water is pumped. Since the stirring of the reactor is assumed to be ideal, it is also assumed to be sufficient to create good contact between the reactor liquid and the tubes. The flow inside the cooling tubes is assumed to be a dispersed plug flow. All symbols used in this and the following sections are defined in Section 7.

A complete state space representation of the resulting model is given in Olesen et al. (2008). However, here the simplifications are made that for each substance, the heat capacity $c_{p}$ and the density $\rho$ are considered constant. These simplifications are motivated in Olesen et al. (2008) and they significantly simplifies the nonlinear simulations. Figure 1 shows a sketch of the tank reactor and a presentation of signals, state variables and some main parameters.

Mass balances for the reactant, the product and for the liquid solvent give the first two state space equation in a representation of the nonlinear tank reactor model:

$$
\begin{aligned}
\frac{d c_{A}}{d t} & =\frac{Q_{\text {in }}}{V} c_{\text {Ain }}-Q_{\text {out }} \frac{c_{A}}{V}-c_{A} k_{0} e^{-\frac{E}{R T}}=f_{1} \\
\frac{d c_{B}}{d t} & =\frac{Q_{\text {in }}}{V} c_{\text {Bin }}-Q_{\text {out }} \frac{c_{B}}{V}+c_{A} k_{0} e^{-\frac{E}{R T}}=f_{2} \\
c_{l} & =\frac{\rho_{l}}{M_{l}}\left(1-\frac{c_{A} M_{A}}{\rho_{A}}-\frac{c_{B} M_{B}}{\rho_{B}}\right)
\end{aligned}
$$

A volume balance gives the outflow $Q_{\text {out }}$ as:

$$
Q_{\text {out }}=Q_{\text {in }}+V c_{A} k_{0} e^{-\frac{E}{R T}}\left(\frac{M_{B}}{\rho_{B}}-\frac{M_{A}}{\rho_{A}}\right)
$$

Using different specific heat capacities for all substances, the heat balance can be written as: 


$$
\begin{aligned}
\frac{d T}{d t} & =\frac{1}{\left(c_{A} c_{p A}+c_{B} c_{p B}+c_{l} c_{p l}\right)}\left[\frac { Q _ { i n } } { V } \left\{c_{A i n} c_{p A}\left(T_{i n}-T\right)\right.\right. \\
& \left.+c_{B i n} c_{p B}\left(T_{i n}-T\right)+c_{l i n} c_{p l}\left(T_{i n}-T\right)\right\} \\
& \left.+c_{A} k_{0} e^{-\frac{E}{R T}}\left(c_{p A}-c_{p B}\right)+\left(-\Delta H_{r}\right) c_{A} k_{0} e^{-\frac{E}{R T}}\right] \\
& -\frac{h_{c} A_{c}}{V}\left(T-T_{c m}\right)=f_{3}
\end{aligned}
$$

A conventional model, found in for example Lagerberg and Breitholtz (1997), is used for the cooling system where the mean temperature of the cooling water $\left(T_{c m}\right)$ is used as a state variable:

$$
\begin{aligned}
\frac{d T_{c m}}{d t} & =\frac{h_{c} A_{c}}{V_{c} \rho_{c} c_{p c}}\left(T-T_{c m}\right) \\
& +\frac{Q_{c}}{V_{c}}\left(1-e^{-\frac{h_{c} A_{c}}{Q_{c} \rho_{c} c_{p c}}}\right)\left(T_{c i n}-T\right)=f_{4}
\end{aligned}
$$

The model has two major nonlinearities: The Arrhenius factor in the reaction rate in (1) and (2) and the exponential term in $(6)$, since $Q_{c}$ is the control signal. However, if $Q_{c}$ is constant and $T_{c i n}$ used as control signal, the exponential term would become a constant and the model would only have one major nonlinearity. Clearly, this is also the case if $T_{c m}$ is directly used as the control input.

\section{LINEARIZED TANK REACTOR MODEL}

The most straightforward approach to achieve a linear control strategy is to linearize the nonlinear model in steadystate operating points. The steadystate operating points of the tank reactor model are given by Equations (1), (2), (5) and (6) set to zero. These four equations then give the steadystate values of four parameters, given values of all other parameters. Here, fixed values of $Q_{i n}$, $c_{A i n}, c_{B i n}$ and $T_{c i n}$ are used and the values of $Q_{c}, c_{A}, c_{B}$ and $T_{c m}$ are calculated for different values of the reactor temperature $(T)$. Hence, the steadystate points are given by the value of the measured output. It should be noted that $Q_{\text {out }}$ is a function of the state variables. Partial derivatives of $Q_{\text {out }}$ have to be taken into account during the linearization, see Olesen et al. (2008) for details. A first order Taylor expansion at the stationary operating points gives the linear model

$$
\begin{aligned}
\frac{d \mathbf{x}}{d t} & =\mathbf{A} \mathbf{x}+\mathbf{B} \Delta Q_{c}+\mathbf{N} \mathbf{v} \\
y & =\Delta T=\mathbf{C x}
\end{aligned}
$$

where

$$
x=\left[\begin{array}{l}
\Delta c_{A} \\
\Delta c_{B} \\
\Delta T \\
\Delta T_{c m}
\end{array}\right] \quad \text { and } \quad \mathbf{v}=\left[\begin{array}{c}
\Delta c_{\text {Ain }} \\
\Delta c_{\text {Bin }} \\
\Delta Q_{i n} \\
\Delta T_{i n} \\
\Delta T_{c i n}
\end{array}\right]
$$

However, during transients the true nonlinear system will not operate near the steadystate operating points. Hence, linearized models, representing the nonlinear system at non-steadystate operating points are needed to more fully describe the nonlinear system. Therefore, the nonlinear model has also been linearized in non-steadystate operating points. These non-stationary operating points used for linearization were determined from simulations of the responses of the nonlinear model to variations in the input signal $Q_{c}$. Linearization in such points results in linearized models with additional constant terms, originating from the non-zero time derivatives of the state variables. Treating these constant terms as input disturbances, the linearized model can be written:

$$
\begin{aligned}
\frac{d \mathbf{x}}{d t} & =\mathbf{A} \mathbf{x}+\mathbf{B} \Delta Q_{c}+\mathbf{N}\left[\begin{array}{l}
\Delta c_{\text {Ain }} \\
\Delta c_{B i n} \\
\Delta Q_{i n} \\
\Delta T_{i n} \\
\Delta T_{c i n}
\end{array}\right]+\frac{d}{d t}\left[\begin{array}{l}
c_{A} \\
c_{B} \\
T \\
T_{c m}
\end{array}\right]_{\mathbf{x}=0} \\
& =\mathbf{A} \mathbf{x}+\mathbf{B} u+\mathbf{N} \mathbf{v}+\varphi \\
y & =\Delta T=\mathbf{C} \mathbf{x}
\end{aligned}
$$

All linearized models, both for steadystate operating points and for non-steadystate operating points, were found observable and stabilizable (see Rugh (1996)).

The stability of the open loop linearized systems depends on the operating point used for the linearization. For some operating points all eigenvalues are stable but a slight change of operating point will give the linearized models one or two unstable eigenvalues. The stability of the linearized models also reflects the local stability of the nonlinear model (see Kahlil (1996)).

\section{QFT ANALYSIS}

Since QFT requires linear control strategies, the nonlinear system described in Section 3 must somehow be translated into a linear system. Horowitz (1993) presented a method based on equivalent linear models for performing QFT design on nonlinear systems. However, finding these equivalent linear models requires knowledge of which input sequence that will give a desired output sequence. This is not trivial, since the nonlinear model is unstable. However, linearized models, describing the tank reactor process near operating points are provided in (9) and (10) and in more detail in Olesen et al. (2008). The models linearized in steadystate operating points, i.e. the models described by Equations (7) to (8), were used as a first attempt to approximate the nonlinear model. Models linearized at temperatures between $300 \mathrm{~K}$ and $450 \mathrm{~K}$ at steadystate operating points are displayed in a Nichols chart in Figure 2 , where the nominal model is chosen at $350 \mathrm{~K}$ and is indicated by a thicker curve.

As seen from Figure 2, the templates will grow large for low frequencies. However, using a controller with integral action, the amplitude of the sensitivity function will be low anyway for these low frequencies. For frequencies, where the amplitude of the sensitivity function may have resonance peaks, the templates are much smaller. An investigation of the corresponding Horowitz-Sidi bounds showed that one single linear controller could be used to control all the models linearized in the stationary operating points. However, when applied to the nonlinear system the control failed even after only small deviations from the equilibrium points. Therefore, in order to describe more of the nonlinear system, models linearized in a number of non-steady-state points were included in the set as well. The new operating points were selected from 


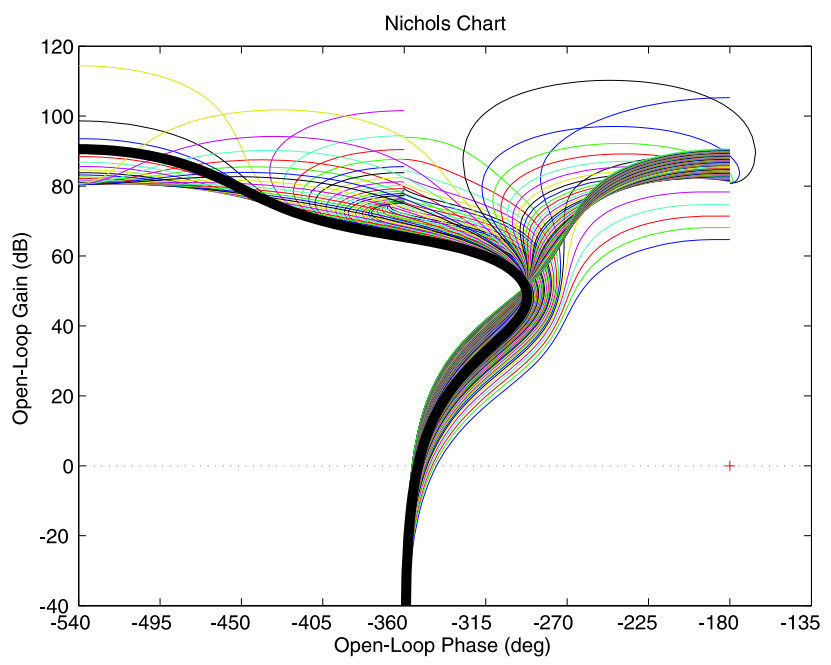

Fig. 2. The models linearized in steadystate operating points

simulations of the nonlinear model. The first non-steadystate points originate from open loop simulations of small steps in the control signal. In the next set of simulations a first attempt of QFT based controllers were used and new operating points were added as points where the controllers could no longer stabilize the system. Models linearized in new operating points were iteratively added to the set of linear models accordingly. In Figure 3 the complex representations of all these new linearized models are plotted in the same Nichols chart as the previous ones, illustrating how the templates become larger. In fact, the resulting Horowitz-Sidi bounds are found to be very hard to fulfill. The closed loop specifications can therefore not be chosen too hard. The closed loop criterion to be fulfilled was chosen as:

$$
\|S\|_{\infty}<6 d B
$$

Even if a linear controller could be designed to fulfill all bounds, the performance would likely not be acceptable. However, if the models are grouped into a few temperature intervals, one higher performing linear controller can be determined for each interval. Five overlapping intervals were found to be satisfactory. In the following subsection, the procedure for finding a suitable controller for low temperatures is described in detail. The remaining four controllers have been developed in the same manner. The issue here is to investigate if a small number of linear controllers can be used. The QFT analysis shows that it is possible to find these controllers. The interval for each controller is decided before the QFT method is used to find an appropriate controller. Hence, in a true system the intervals can be chosen such that the most likely operating region will fall entirely within the interval of one single controller. The number of overlapping intervals can also be decided upon when using this method on a true system. Smaller intervals will give a larger number of controllers, each one of them more optimal for control of the nonlinear system within the interval. When implementing the overlapping controllers found from the QFT procedure, the controller switching should be performed by some standard method applying bumpless transfer and for example hysterisis to avoid sliding mode.

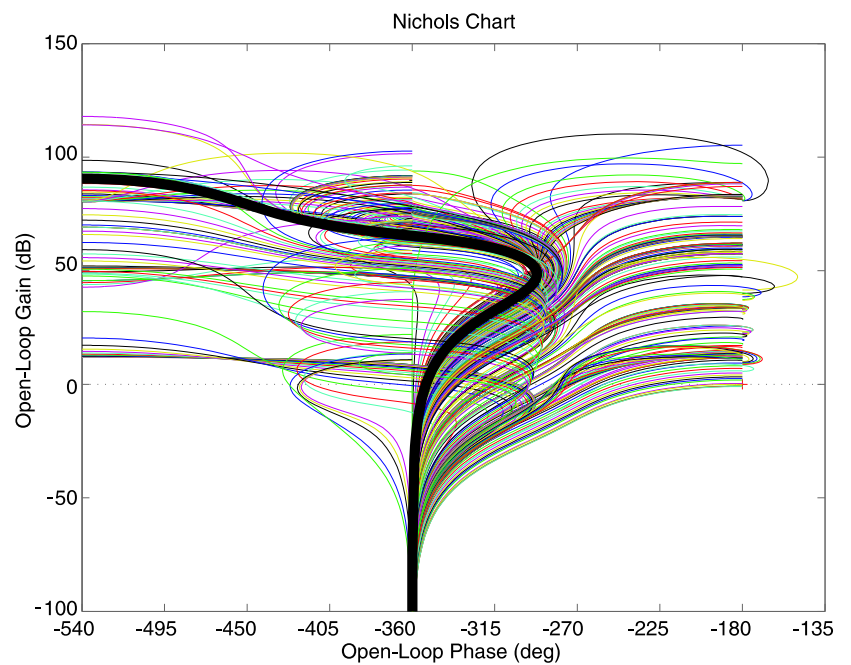

Fig. 3. Models linearized in operating points and in a number of other points

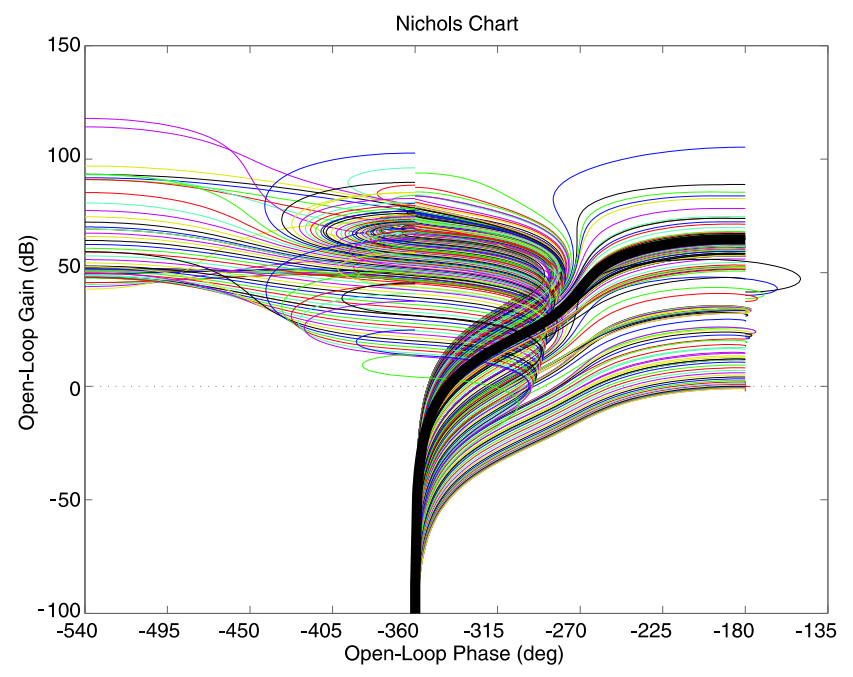

Fig. 4. Low temperature linearized models.

\subsection{QFT for Low Temperatures}

As an example of the QFT based design, the temperature interval $300 \mathrm{~K}$ to $330 \mathrm{~K}$ is chosen. First, the size and shape of the templates are investigated. Figure 4 shows the complex representations of the linearized models in a Nichols chart. The nominal model chosen is the model linearized in the steadystate operating point where $T=$ 300 .

None of the linear open loop models is allowed inside the $6 d B$ curve of the Nichols chart at any frequency. A selection of the resulting Horowitz-Sidi bounds for the nominal model to stay outside is shown in Figure 5. In Figure 5, where the Nichols diagram of the nominal model is shown as a thick, solid curve to the left. As a first attempt, a controller suitable for control of all models linearized in the stationary operating points is used:

$$
F_{y 1}=-0.0008 \frac{(s+0.06)(s+0.0008)}{s(s+0.3)(s+0.04)}=-0.0008 F_{y}
$$

The Nichols diagram of the nominal open loop model in series with $F_{y 1}$ is shown in Figure 5 as the solid thick 


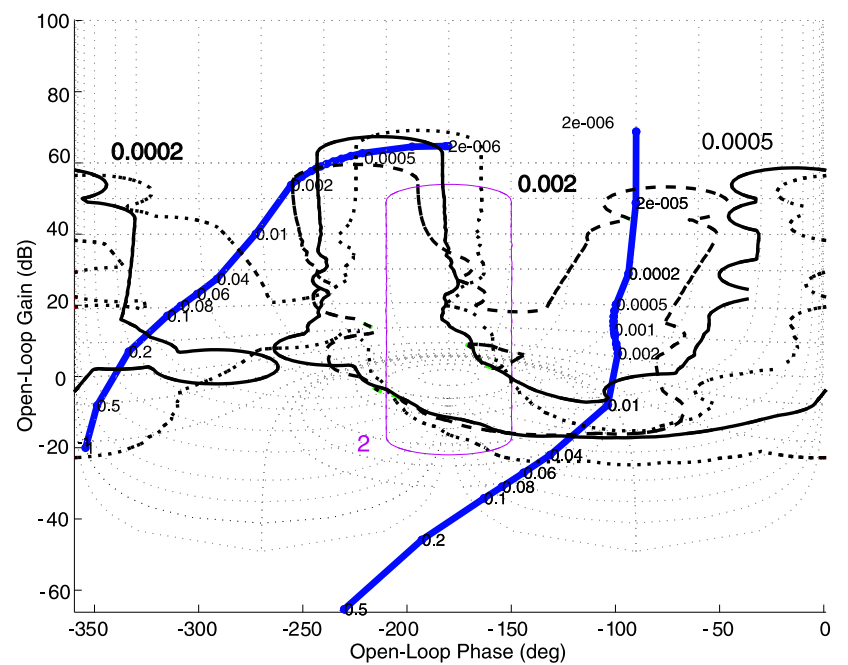

Fig. 5. A selection of Horowitz-Sidi bounds for frequencies 0.0002 (dotted), 0.0005 (solid), 0.002 (dashed) and 2 (thin solid). The nominal model (left, thick) and nominal model in series with a preliminary controller $F_{y 1}$ (right, thick)

curve to the right. As can be seen from the figure, the open loop models using the controller $F_{y 1}$ will violate the Horowitz-Sidi bounds. One way of solving that problem is to lower the high frequency gain of the controller. Yet another possibility is to increase the gain, use a lead filter to get a phase lift at $\omega=5 \cdot 10^{-4}$ and a filter for loopshaping at high frequencies. An example of that kind of controller is:

$F_{y 2}=-75 F_{y} \frac{(s+0.6)(s+0.2)(s+0.015)\left(s+10^{-4}\right)}{(s+1.5)(s+0.025)(s+0.01)}$

Stability The criterion (11) is not sufficient to guaranty stability of all closed loop models. Hence, a stability criterion must be used in addition to (11). Since most linearized models are unstable, the simplified Nyquist theorem cannot be used to verify stability of the closed loop models. Thus, the characteristic equations $1+L_{i}(s)=$ 0 have been calculated for all plant cases to sort out cases where roots of the characteristic equation have positive real part. Adding the stability criterion to the closed loop specifications shows that lowering the high frequency gain will result in unstable closed loops for a group of linear open loop unstable models within the set of models investigated. Therefore, the above procedure using loop shaping and a lead filter was used, which resulted in the controller (14). With this controller the nominal open loop model stays well outside all Horowitz-Sidi bounds as shown in Figure 6.

Simulations of the nonlinear tank reactor model in closed loop with this controller shows that as long as the cooling system has not reached its physical limitations, the controller performs very well in reducing the impact of input disturbances. The servo problem has not been the issue here, though the controller is found to be able to follow step reference changes up to $20 \mathrm{~K}$.

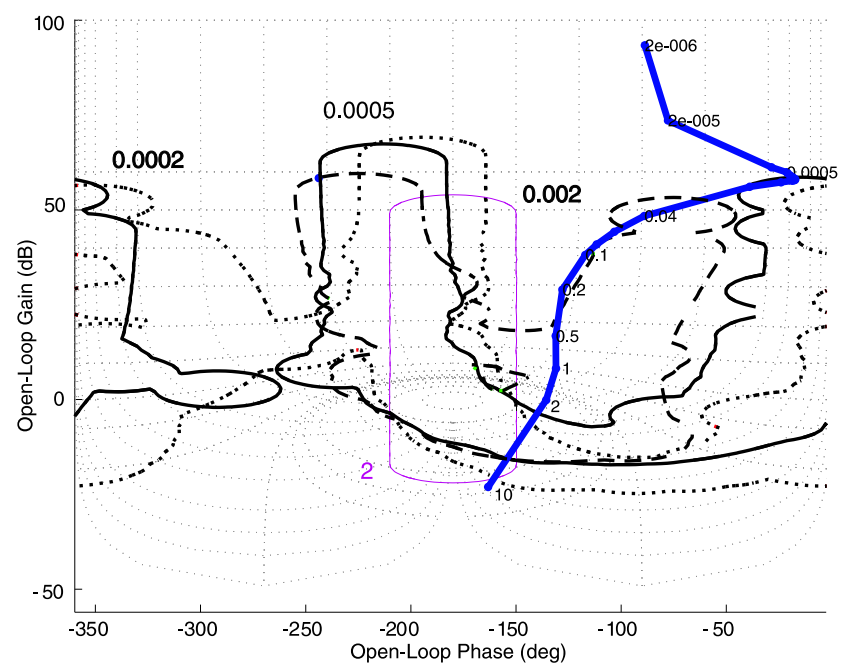

Fig. 6. A selection of Horowitz-Sidi bounds and the nominal model in series with a better controller $F_{y 2}$

\section{CONCLUSIONS}

Quantitative feedback theory (QFT), a theory for linear uncertain systems, has been used for designing temperature control of an ideally stirred tank reactor operated continuously with an exothermic reaction. This system is highly nonlinear and locally unstable. Linearizing the model in a number of operating points gives a set of linear models that is regarded as an uncertainty description. The QFT method shows that if the reactor is operated very close to the stationary linearization points one rather simple linear controller can be used for the whole operating window. However, control of the nonlinear model sometimes requires operation outside a stationary point which this first controller cannot handle. Models linearized in relevant non-stationary operating conditions were therefore added to the uncertainty description to improve the robustness. Further, the operating window was divided into a low number of temperature intervals, each having a designated controller. In this paper five linear controllers have been found where each one of them performs very well in simulations with the nonlinear model. The QFT method provides a framework for finding a linear control structure, that is intuitive to use. However, there are no optimal solutions provided by the method and the design can be improved indefinitely. The controller design of this example can be improved further using the method of finding equivalent linear models, described in Horowitz (1993), now that suitable input signals are provided by the controllers designed here. 


\section{LIST OF SYMBOLS}

A The reactant

$A_{c} \quad$ Area of cooling system $\left(m^{2}\right)$

$B$ The product

$c_{\lambda} \quad$ Concentration of substance $\lambda=A, B, l(\mathrm{~mol})$

$c_{\lambda i n}$ Feed concentration of substance $\lambda\left(\mathrm{mol} / \mathrm{m}^{3}\right)$

$c_{p, \lambda}$ Heat capacity of substance $\lambda(J /(\mathrm{mol} \cdot K))$

$c_{p c}$ Heat capacity of cooling liquid $(J /(\mathrm{mol} \cdot K))$

$E$ Activation energy $(\mathrm{J} / \mathrm{mol})$

$h_{c} \quad$ Heat transfer coefficient from cooling system to reacting liquid $\left(W /\left(K \cdot m^{2}\right)\right)$

$k_{0} \quad$ Reaction rate coefficient $\left(s^{-1}\right)$

$l \quad$ Liquid solvent

$M_{\lambda} \quad$ Molar mass of component $\lambda(\mathrm{kg} / \mathrm{mol})$

$Q_{\text {in }}$ Feed flow to the reactor vessel $\left(\mathrm{m}^{3} / \mathrm{s}\right)$

$Q_{\text {out }}$ Outlet flow from the reactor vessel $\left(\mathrm{m}^{3} / \mathrm{s}\right)$

$Q_{c} \quad$ Feed flow to the cooling system $\left(\mathrm{m}^{3} / \mathrm{s}\right)$

$r \quad$ Reaction rate $\left(\mathrm{mol} /\left(\mathrm{m}^{3} \cdot \mathrm{s}\right)\right)$

$R \quad$ The ideal gas constant $(J /(\mathrm{mol} \cdot K))$

$t \quad$ Time $(s)$

$T$ Temperature in the reactor $(K)$

$T_{c m}$ Mean temperature in the cooling system $(K)$

$T_{\text {cin }}$ Inflow temperature to the cooling system $(K)$

$V \quad$ Volume of the reactor vessel $\left(\mathrm{m}^{3}\right)$

$V_{c} \quad$ Volume of the cooling system $\left(\mathrm{m}^{3}\right)$

$\Delta H_{r}$ Heat of reaction $(\mathrm{J} / \mathrm{mol})$

$\rho_{\lambda} \quad$ Density of component $\lambda\left(\mathrm{kg} / \mathrm{m}^{3}\right)$

$\rho_{c} \quad$ Density of the cooling liquid $\left(\mathrm{kg} / \mathrm{m}^{3}\right)$

\section{REFERENCES}

P-O. Gutman. Qsyn - the Toolbox for Robust Control Systems Design for use with Matlab, User's manual. NovoSyn AB, Sweden, 1996.

M.A. Henson and D.E. Seborg, editors. Nonlinear process control. Prentice-Hall PTR, Upper Saddle River, 1997.

I.M. Horowitz. Quantitative Feedback Design Theory (QFT). QFT Publications, Boulder, Colorado, 1993.

H.K. Kahlil. Nonlinear Systems. Prentice Hall, Upper Saddle River, New Jersey, 1996.

A. Lagerberg and C. Breitholtz. A study of gain scheduling control applied to an exothermic CSTR. Chemical Engineering 8 Technology, 20:435-444, September 1997.

J.D. Morningred, B.E. Paden, D.E. Seborg and D.A. Mellichamp. An adaptive nonlinear predictive controller. Chemical Engineering Science, 47:755-762, March 1992.

V. Olesen, C. Breitholtz and T. Wik. Closed Loop Model Reduction Applied to a Tank Reactor Process. Chemical Engineering Science, 63:674-684, February 2008.

V. Olesen, T. Wik and C. Breitholtz. A Closed Loop Approach to Tank Reactor Model Simplification. Proceedings of the 16th IFAC World Congress, Prague, Czech Republic (P. Horacek, M. Simandl and P. Zitek, editors), 2005.

W.J. Rugh. Linear System Theory. Prentice Hall, Upper Saddle River, New Jersey, 1996.

L.P. Russo and B.W. Bequette. Effect of process design on the open-loop behavior of a jacketed exothermic CSTR. Computers and Chemical Engineering, 20:417426, April 1996.
T. Zhang and M. Guay. Adaptive control of uncertain continously stirred tank reactors with unknown actuator nonlinearities. ISA Transactions, 44:56-686, 2005. 